\title{
How physicians can empower patients with digital tools
}

\section{A joint study of the Italian Scientific Society of Internal Medicine (FADOI) and the European Federation of Internal Medicine (EFIM)}

\author{
Letizia Affinito $^{1}$ (D) $\cdot$ Andrea Fontanella $^{2} \cdot$ Nicola Montano $^{3} \cdot$ Antonio Brucato $^{4}$
}

Received: 22 April 2020 / Accepted: 10 July 2020 / Published online: 23 July 2020

(C) The Author(s) 2020

\begin{abstract}
Background While there is broad consensus that the use of digital tools would significantly improve patient empowerment, to date, an improvement in health outcomes has been elusive.

Objective The objective of this study was to assess how to improve the ability of physicians to empower patients with digital tools.

Methods We conducted a web-based survey using SurveyMonkey over nearly 6 months. A questionnaire was sent with an email, explaining the aims of the survey and providing a link to complete the web-based questionnaire, to the heads of each of the 37 medical national societies adhering to the EFIM (European Federation of Internal Medicine), inviting them to disseminate the questionnaire among their members.

Results Two hundred and eighteen responses were received. They suggest that the main success factors in increasing and improving patient empowerment with digital tools and realizing health goals are clinical evidence, followed by patient/ physician involvement in the design, tools designed around the real needs of the patient, and reimbursement. Most of the respondents who have already prescribed digital tools for patient empowerment are just enough satisfied with the results achieved by their patients. Interestingly, $18 \%$ of the respondents had spent more than $30 \mathrm{~min}$ on the visit of patient to doctor. However, the majority devoted only 5-9 min to illustrating the suggested digital tools.

Conclusions According to the respondents, clinical evidence, motivation, physician and patient's involvement in design, and reimbursement, as well as organizations' appropriate business models and support, are the main determinants of the diffusion and effective adoption of digital tools for successful patient empowerment in internal medicine.
\end{abstract}

Keywords Empowerment $\cdot$ Adoption $\cdot$ Patient centricity $\cdot$ Patient communication $\cdot$ Human-centered design

\section{Introduction}

The term 'patient empowerment' is increasingly used to describe situations where patients are encouraged to

Letizia Affinito

laffinito@letiziaaffinito.com

1 Boston College, Boston MA 02467, MA, USA

2 Department of Medicine, Buon Consiglio-Fatebenefratelli Hospital, Naples, Italy

3 Department of Clinical Science and Community Health, Universita' degli Studi di Milano, Milan, Italy

4 Department of Biomedical and Clinical Science, University of Milano, Fatebenefratelli Hospital, Milan, Italy proactively manage their own health. It is increasingly considered as critical to improve the quality of healthcare, but leads inevitably to the question of how patient empowerment can be successfully achieved?

Nowadays, apart from psychological implications, patient empowerment in everyday practice depends on digital tools and how they are used.

Indeed, one question is often raised by healthcare managers and/or HCPs (healthcare professionals): Is digital health technology effectively empowering patients?

The key question behind our survey was "how can physicians empower their patients if they themselves are not being effectively empowered by their organizations or other stakeholders?"

This study provides a new perspective on the adoption of digital tools to empower patients. It differs from most previous 
surveys. They have mainly focused on exploring the increased use of digital tools to empower patients, or the main barriers to the adoption of technologies by physicians. In contrast, our survey focuses on the actual experience of physicians with digital tools, and on their potential impact on both patient empowerment and clinical practice as well as healthcare outcomes. Most importantly, it builds on the experience of physicians to identify both critical success factors and possible approaches to effectively empower physicians and patients to improve healthcare outcomes as a result. The underlying assumption is that investing in digital tools alone and focusing exclusively on patient empowerment is not enough to move the needle to realize patients' health goals.

Our survey derives from a literature review conducted between 2012 and 2018 to assess the state-of-the-art knowledge and understanding of patient empowerment in the digital era, its characteristics, and its impact on both patients' health and clinical practice and healthcare outcomes.

We focus on internal physicians because we feel that with the advent of new media and digital tools in healthcare management, they are positioned as the primary reference figure to lead the change towards optimal citizen/patient empowerment. In fact, the internal physician:

1. treats patients affected by a wide variety of disorders (e.g., hepatological, cardiological, endocrinological, oncological, autoimmune disorders, allergological, gastroenterological, rheumatological, etc.).

2. assesses the patient's health in its entirety and complexity, considering both the physical and psychological or social aspects, and then prescribing any diagnostic tests, making a diagnosis and prescribing a drug therapy or, if necessary, directing the patient to a more suitable specialist in the sector

3 . is the specialist of the complex patient with polypathology, able to establish a list of priorities among the various coexisting diseases, and to know the influences of one pathological picture on another and the pharmacological interactions of the polytherapy.

This is why we partnered with FADOI-EFIM. FADOI is the Italian Federation of the Associations of Hospital Internal Physicians. EFIM is the European Federation of Internal Medicine which was formed by bringing together the national societies of internal medicine from each of the European countries, both inside and outside the European Union.

We structured the research around the following building pillars derived from the literature review:

\section{A shared definition of patient empowerment}

Probably one of the first and main issues when dealing with patient empowerment is the multiple ways in which it is defined, not only depending on the context and objectives but also on the culture, mindset, and background of each stakeholder.

To get started, let us clarify the issue of "power". Some authors claim that empowerment has nothing to do with power, thus, nothing to do with giving or taking power (Anderson and Funnell 2010). On the other hand, others such as Laverack (2006) or Monteagudo Peña and Gil (2009) think that power is at the core of the concept of empowerment. As pointed out by Page and Czuba (1999), empowerment is not possible if power is inherent in positions or people. Consequently, it is fundamental to expect its shift from one person or group to another. It is also crucial to note that power is not only having the necessary knowledge, but also being able to exercise a choice based on that knowledge (Laverack 2006). If a given knowledge is not applicable, the person may feel even more powerless.

We feel that empowerment has nothing to do with power and much more to do with responsibility, trust, and a sense of control over a disease.

Taking a closer look at the notion of patient empowerment as evidenced in the scientific literature, patient empowerment is generally used to refer to the control of patients over their health and condition, as well as their ability to be more involved in their healthcare.

Gibson, in a review about patient empowerment in health, redefined empowerment as a process of helping people to assert control over the factors which affect their health (Gibson 1991).

Another literature review (Calvillo et al. 2013) defines patient empowerment as a continuous process through which patients (and patient groups) work in partnership with their healthcare system.

The objective of this collaboration is to enable patients to become more responsible for and involved in their treatment and healthcare.

For the aim of this survey we refer to the definition provided by the WHO, which defines empowerment as "a process through which people gain greater control over decisions and actions affecting their health" and which should be seen as both an individual and a community process.

Four components have been reported as being fundamental to the process of patient empowerment: 1) understanding by the patient of his/her role, 2) acquisition by patients of sufficient knowledge to be able to engage with their healthcare provider, 3) patient skills, and 4) the presence of a facilitating environment.

Based on these four components, empowerment can be defined as: A process in which patients understand their role, are given the knowledge and skills by their healthcare provider to perform a task in an environment that recognizes community and cultural differences, and which encourages patient participation (World Health Organization 2009). 
The best way to define patient empowerment would be to define it as an embracing practice that motivates patients to be mindfully involved in their providers' care services. The final goal of empowering patients is to guide them in the development of self-awareness and self-care while promoting patients as equal partners in their healthcare decisions.

In the face of different definitions, the shared core idea about patient empowerment depicts it as an attempt for patients to take charge of their own health.

\section{The use of digital technologies to empower patients}

Nowadays, beyond cognitive connotations, technology and the way it is used are central in the empowerment of patients, mostly when they are affected by a chronic disease.

Mahmood et al. (2019) found that compared to those without mobile health (mHealth) apps, individuals with mHealth apps had significantly higher odds of using their smart devices to track progress on a health-related goal [adjusted odds ratio (aOR) 8.74, 95\% confidence interval (CI): 5.66-13.50, $P<.001)]$, to make a health-related decision (aOR 1.77, 95\% CI: $1.16-2.71, P<.01)$, and to engage in healthrelated discussions with care providers (aOR 2.0, 95\% CI: 1.26-3.19, $P<.01$ ) (Mahmood et al. 2019).

In their study, Milani et al. (2017) highlights how a digital hypertension program is feasible and associated with significant improvement in blood pressure control rates and lifestyle change (Milani et al. 2017).

Indeed, digital health technology is currently not always effectively empowering patients because of the several barriers identified both among physicians and in the healthcare system.

Based on a study by Cahn et al. (2018), digital diabetes care has demonstrated only modest $\mathrm{HbA} 1 \mathrm{c}$ reduction in multiple studies and borderline cost-effectiveness, although patient satisfaction appears to be increased. Better understanding of the barriers to digital diabetes care and identification of unmet needs may yield improved utilization of this evolving technology in a safe, effective, and cost-saving manner (Cahn et al. 2018).

In a study conducted by Sleurs et al. (2019), 112 apps were retained for analysis and could be classified in five categories: asthma $(n=71)$, COPD $(n=15)$, asthma and COPD $(n=15)$, rhinitis and asthma $(n=5)$, and rhinosinusitis $(n=6)$. Eighty percent were developed by medical technology companies ,compared to $18 \%$ by medical doctors and $2 \%$ by pharmaceutical companies. Two-thirds of apps allow disease self-monitoring, whereas over half of apps provide patient feedback through graphs. Sixty percent of apps contain easily accessible patient education material. Only $3 \%$ of apps reach a score of $\geq 7$ on the newly designed patient empowerment index (Sleurs et al. 2019).
The critical role of technology use in the empowerment of patients is strongly supported by the most recent definition of "digital health" shared by the Healthcare Information and Management Systems Society (HIMSS), suggesting that "Digital health connects and empowers people and populations to manage health and wellness, augmented by accessible and supportive provider teams working within flexible, integrated, interoperable, and digitally-enabled care environments that strategically leverage digital tools, technologies, and services to transform care delivery."

A variety of digital tools are currently available to help patients manage their health care and receive the services they need:

Telehealth: the use of digital information and communication technologies, such as computers and mobile devices, to access healthcare services remotely and manage patients' healthcare. These may be technologies patients use from home or that their doctor uses to improve or support healthcare services. The following examples of telehealth services may be beneficial for patients' empowerment:

Patient portal: Provided by primary care clinics, patient portals offer an alternative to email, which is a generally insecure means to communicate about private medical information. A portal provides a more secure online tool to do the following:

- Communicate with a doctor or a nurse.

- Request prescription refills.

- Review test results and summaries of previous visits.

- Schedule appointments or request appointment reminders.

If a doctor is in a large healthcare system, the portal also may provide a single point of communication for any specialists a patient may see.

Remote monitoring: a variety of technologies enabling a doctor or healthcare team to monitor patients' health remotely. These technologies include:

- Web-based or mobile apps for uploading information, such as blood glucose readings, to your doctor or healthcare team.

- Devices that measure and wirelessly transmit information, such as blood pressure, blood glucose, or lung function.

- Wearable devices that automatically record and transmit information, such as heart rate, blood glucose, gait, posture control, tremors, physical activity, or sleep patterns.

- Home monitoring devices for older people or people with dementia that detect changes in normal activities such as falls.

Personal health records (PHR): a collection of information about patients' health that he/she control and maintain. A PHR 
app is accessible to patients anytime via a web-enabled device, such as a computer, laptop, tablet or smartphone.

Personal health apps: they help patients better organize their medical information in one secure place. These digital tools may help patients:

- Store personal health information.

- Record vital signs.

- Calculate and track caloric intake.

- Schedule reminders for taking medicine.

- Record physical activity, such as your daily step count.

Based on a literature review of 266 papers in 2013, Calvillo et al. (2013) highlight that there is a wide spectrum of technologies empowering patients (Calvillo et al. 2013). There are initiatives that use promising technologies (such as games and virtual worlds or textile monitoring), and others reuse traditional technologies (e.g., audio call or video recording).

Web services and communication networks have been reported as being the most used technologies ( 74 and 51 articles respectively) to make remote communication and access to health information and services easier (Calvillo et al. 2013).

Aside from them, both personal health record (PHR) and electronic health record (EHR) approaches share superior positions. Very similar to each other, they empower patients in different ways. By using EHR the patient can have access to and knowledge of his/her health information, while with PHR he/she is also granted administration privileges. Jorge Calvillo et al.'s literature review (Calvillo et al. 2013) shows a slightly higher use of PHR (12.7\% of articles) for empowering patients than of EHR (10\%).

Additional interesting ways to empower patient focus on translating the methodology of patient support groups to the virtual world, using social media and online communities where the patient receives advice from peers and he/she can provide information for others.

Finally, other relevant technologies include the internet as a source of information, software and mobile apps, security mechanisms, devices, and communication media (such as traditional and IP telephony or e-mail).

Having identified the technologies mostly used to empower patients, it is crucial to keep in mind that the same technology can deploy two different approaches for patient empowerment. For example, e-mail communication could be used for strengthening doctor-patient relations or alerting a patient of modifications to his/her health information record. The most popular route to empowerment is patient education - shared by $40 \%$ of reviewed articles. It is widely argued that an educated patient can make more informed decisions, improve compliance, reduce anxiety levels, and participate actively in the treatment of his/her diseases. This fact is more relevant in chronic scenarios where the patient must modify his/her life and adapt to permanent conditions. If healthy scenarios were considered, the benefits of patient education could be translated to the maintenance of health and prevention tasks through citizen education.

From Jorge Calvillo et al.'s literature review (Calvillo et al. 2013), we can conclude that different levels of empowerment exist. All the reviewed approaches have the same objective (i.e., to empower patients), but the grade of autonomy or involvement that the subject obtains varies from one solution to another.

Obstacles to effective patient empowerment abound. First, in order to play their proactive roles, patients must able to trust in the technology empowering them. Second, another critical obstacle is the reluctance of doctors to lose their power.

Thus, involved actors' attitudes towards patient empowerment will determine the real speed of delivery of healthcare delivery models and the role of each actor. If obstacles and gaps are successfully addressed, technology will, in the medium term, enable the emergence of a new type of patient fully equipped for the challenging healthcare scenarios of the $21 \mathrm{st}$ century.

\section{Patient empowerment as a process}

Empowerment, by definition, is a social process, since it occurs in relationship with others. It is a process similar to a path or journey, one that develops as we work through it. Aujoulat et al. (2008) define patient empowerment as a process.

Other authors have described patient empowerment as both a process and an outcome. For instance, Funnell et al. (1991), emphasized that while we can see empowerment as "the process of discovery and development of one's inherent capacity to be responsible for one's life", they pointed out that "patient empowerment is fundamentally an outcome". Therefore, patients are empowered "when they have the knowledge, skills, attitudes, and self-awareness necessary to improve the quality of their lives".

Anderson and Funnell (2010) define patient empowerment as "a process designed to facilitate self-directed behavior change".

\section{Physicians play a critical role in patient empowerment}

While patient empowerment is increasingly considered as critical to increase healthcare outcomes, it is still unclear how patient empowerment will be successfully achieved and how digital health technology can effectively empower patients. Most importantly, what is the physician's role, and are there a number of variables which are not in the hands of the physicians that need to be identified and managed by both physicians and non-physicians for patient empowerment in order to produce the expected outcomes? 
With this aim, let us examine the psychology behind patient empowerment and the role of physicians.

Patients are constantly making decisions with regard to care. It begins with how they interpret symptoms, such as whether that persistent pain is circumstantial or worthy of medical attention. In deciding treatment options, a patient makes the most explicit choice based on information they have gathered. Finally, there is the treatment phase where a patient must be committed to treatment (Shahin 2008). This means that there are three characteristic situations where a patient must make decisions: observation of state of health before presenting to a HCP, discussion and decision during the medical interview, and the treatment phase.

Specifically related to the role of the physician for patient empowerment are the discussion and decision during the medical interview, and the treatment phase.

A patient's decisions during the medical interview involve two components. In the first, a patient and their HCPs discuss the medical elements of the condition, as well as treatment options and risks. Essentially, this phase can be considered one of information gathering and is similar to the observation phase, though ideally it is less autonomous, as it is a process done in partnership with the guidance of an HCP.

The decision-making process can be influenced by several biases such as the focusing effect [defined as "not taking into account alternatives to an option that has been initially proposed or generated" (Del Missier et al. 2006)], loss aversion (which refers to the concept that people are more motivated to avoid losses than pursue equal gains, i.e. when considering serious risks from surgery), and availability heuristic, which is employed when a person "estimates frequency or probability by the ease with which instances or associations could be brought to mind" (Tverksy and Kahneman 1973). As with loss aversion, it remains the responsibility of HCPs to notice these heuristics and manage them. This takes time and will probably cause tensions in the patient-HCP relationship, but it is nonetheless a necessary component of good patientcentered healthcare. To counter the focus effect, the exploration of alternative options must be facilitated under the guidance of HCPs.

Finally, a patient must make decisions at the treatment stage. The compliance of patients to the treatments prescribed is unfortunately often very low. While non-compliance is brought about by poor education wth regard to the disease and its treatment timeline, it is made worse by the fact that patients may deny that they are sick or be overconfident regarding their health. These are HCP-independent processes, and are a good argument for increasing the frequency of HCP-patient interactions, discussions about patients' concerns and beliefs, and patient education. For treatments that take place over extended periods of time, particularly those that include asymptomatic phases, patients must be closely monitored to facilitate discussion with HCPs. Unfortunately, many of these thoughts and emotions are tied to external perceptions and stigma, meaning that the scope of the education efforts is widened from being patient-specific to communitywide.

Having clarified the psychology behind patient empowerment, we might say that the role of physicians is to put the patient's specific case in context and to rank choices in a realistic way; to balance risk and benefit.

Decision-making and independence are absolute requirements for the patient. Still, the physician needs to educate, support, and always stay near, ready to advise or catch the patient if they begin to fall.

\section{Physician's empowerment faces several critical barriers}

Despite studies indicating the benefits from certain kinds of e-health technology and interest from policy makers in implementing the innovative technology, the uptake and adoption of e-health technologies has not always been consistent within healthcare practice. The result is that the adoption of these technologies has lagged behind expectations (Ajami et al. 2011). The acceptance by physicians of digital technology is critical, and thus it is important to identify influences that slow the uptake in order to overcome them.

Based on the results of a scoping review carried out by de Grood et al. (2016), a number of barriers and facilitators to the adoption of e-health technology by physicians can be identified.

Among these, threatened clinical autonomy, cost and liability issues, training, and support were the most cited.

Walter and Lopez found that threatened professional autonomy negatively affected perceived usefulness and the intention to use e-health technology (Walter and Lopez 2008). They defined professional autonomy as "professionals" having control over the conditions, processes, procedures, or content of their work according to their own collective and, ultimately, individual judgment in the application of their profession's body of knowledge and expertise professional privacy. De Grood et al. (2016) found that cost and liability issues were associated with the adoption of e-health technology by physicians. Concerns regarding reimbursement were the most cited within this theme. Physicians were less willing to utilize ehealth technology with no reimbursement initiatives present.

As previously anticipated, researchers and policymakers recognize the importance of having an evidence-based measurement of patient engagement, as it is a necessary tool for planning and implementing initiatives. However, with very few studies and limited data available, there is a lack of clearly defined evidence-based guidelines available. This represents a critical barrier to adoption by physicians. A 2013 review (Goldzweig et al. 2013) (including 14 randomized controlled trials) found that "there was no consistent evidence that access 
to a patient portal significantly improved clinical outcome, satisfaction, or adherence to treatment".

Based on the results of the Web in Salute - FADOI survey (2018), physicians are not sure or do not think that digital tools used for patient empowerment reduce the time of the medical interview or improve healthcare outcomes. At the same time, most of the respondents agree that digital tools can improve healthcare outcomes. Besides, physicians reported that the main barriers to successful patient empowerment with digital tools are the lack of clinical evidence or a certification from an independent scientific society or institution.

\section{Objectives}

The primary objectives of our study were to:

- Evaluate the degree to which internal physicians prescribe and use technology to empower patients.

- Evaluate the current degree to which physicians enable patient empowerment.

- Identify barriers to technology use in order to foster patient empowerment.

Secondary objectives included:

- Stimulate physicians' thought about their role in fostering patient empowerment.

- Offer food for thought for the development of initiatives, tools, and services that allow the doctor to become the protagonist of change and offer citizens/patients the opportunity to be adequately involved in clinical decisions concerning their health.

- Generate physicians' engagement and commitment.

\section{Research questions}

1. How is the use of technology affecting patient empowerment?

2. What are the main barriers to effectively foster patient empowerment?

3. How can physicians contribute to effective patient empowerment?

\section{Research methodology}

Questionnaire development We designed the survey to gather data on health digital tools experiences resulting from hospital ambulatory visits with internal physicians.
We focused on visits during which a digital tool had been prescribed and used by physicians to empower their patients (Fig. 1).

We conducted a web-based survey using SurveyMonkey over nearly 6 months. A questionnaire was sent by email with a text explaining the aims of the study and providing a link to complete the web-based questionnaire, to the heads of 37 national societies from 35 member countries adhering to FADOI-EFIM, inviting them to disseminate the survey among their national membership. We received a total of 218 responses.

The web-based survey was closed, voluntary, and took only $13 \mathrm{~min}$ to complete. Free participation to the FADOI National Congress was given as incentive. The SurveyMonkey web-based survey service was used to design the questionnaire, manage the survey, and collect data.

The web questionnaire was eight pages long, and all data were anonymized and protected by Norton and TRUSTe.

Reminder emails were sent after 3, 5, and 7 weeks. The questionnaire was organized in a series of linked pages (multiple-item screens) with electronic instructions to facilitate the flow. A progress indicator was permanently visible.

According to the "Checklist for Reporting Results of Internet E-Surveys" (CHERRIES), all the questions, except the last one, were mandatory to obtain more solid data on the endpoints of the survey. To guarantee the possibility of answering, most questions provided a nonresponse option, that is, "I don't know".

We analyzed only completed questionnaires, excluding questionnaires that had missing data due to the responder stopping early and leaving the website.

If all answers were not completed, it was not possible to continue and confirm the questionnaire. A "back button" was provided to change answers before submitting them, but thereafter, no further changes were allowed. The answers were collected automatically in the SurveyMonkey database.

\section{Development of the questionnaire}

We conducted a literature review in PubMed using the following keywords: "digital technologies," "survey,"

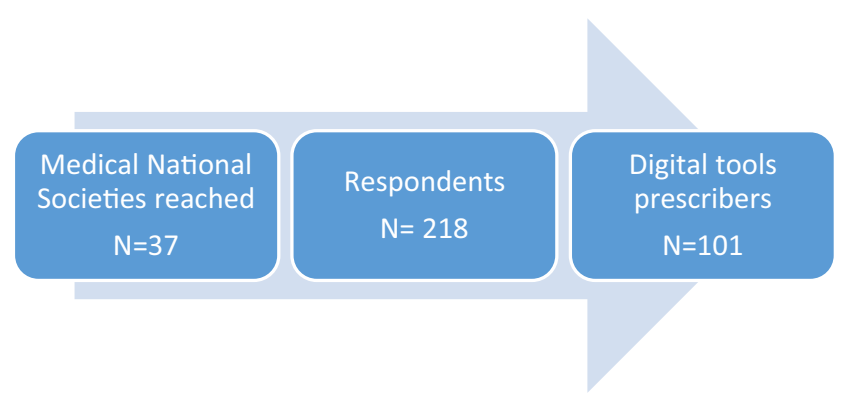

Fig. 1 Study design 
"questionnaire," "eHealth," "mHealth," "digital health," "patient empowerment," and "physician empowerment." We retrieved a number of surveys about "patient empowerment with digital technologies" involving patients but none involved physicians' empowerment. Based on the material collected, we drafted 48 questions organized in the following sections:

- Sources of information used by patients/citizens

- Discussion with patients on the use of digital tools for health management

- Most recent visit

- Awareness

- Engagement

- Empowerment

- Clinical practice information

All respondents, regardless of their use/prescription of digital tools for patient empowerment, were asked about accuracy of information shared by their patients, main sources used to search health information and their use/experience with digital tools, health status, presence of chronic illnesses, recommendations, and socio-demographic characteristics (sex, race/ethnicity, education, insurance status, and drug coverage).

To make sure we only considered those physicians prescribing tools for patient empowerment, if the answer to the question regarding the prescription of digital tools was "no" we redirected respondents to the final section of the questionnaire (Clinical practice information).

A series of questions addressed actions taken during the visit. We asked what was the duration of the visit, the time spent illustrating their prescription/advice, the time spent exploring patients' health experience and their needs, whether the physician wrote a prescription for a tool/digital tool to empower his/her patient, and/or distributed and illustrated educational material to help patients best use the prescribed digital tool to foster their empowerment. Finally, we asked questions about physicians' experience with digital tools and the main barriers to the optimal use of digital tools to empower patients.

We pre-tested the questionnaire with the collaboration of five members of FADOI-EFIM to assess its readability, clarity, and completeness, and to collect suggestions. All members answered with comments and contributions, and a general positive consensus was gathered; hence, the final questionnaire was finalized considering the comments and contributions received.

\section{Recruitment}

Each head of the 37 national societies from 35 member countries adhering to FADOI-EFIM used their databases to reach their own members.
We sent one email invitation to each national society, usually to the president or a member of the board who sent the questionnaire to their members.

The survey was announced on the FADOI and EFIM websites, by email and through the EFIM newsletter.

\section{Results}

The survey was open between April 2019 and October 2019. A total of 218 answers were collected. Responding physicians were mostly from Italy (49\%), Poland (17\%), Switzerland (10\%) and Argentina (10\%), of which $73 \%$ were specialized in internal medicine practicing in public/teaching hospitals $(48 \%)$ with $80 \%$ of the patients in the national health system. Most of the patients (77\%) were affected by a chronic disease (Table 1) with $72 \%$ in a good health status (Fig. 2).

\section{Use and prescription of digital health instruments}

Out of 101 respondents, only $27 \%$ declared that, in the past week or last month, they had prescribed/suggested an application for the management of their pathology, and $23 \%$ a dedicated portal (Fig. 3). When asked "Which tools did you use to actively involve your patient in managing and treating her/his health?" most respondents out of $75(91 \%)$ reported using "dialogue" followed by e-mail (43\%), WhatsApp $(37 \%)$, and paper information material $(33 \%)$.

Table 1 Patients' chronic diseases

\begin{tabular}{lll}
\hline Allergy & $4.40 \%$ & 7 \\
Anxiety & $13.84 \%$ & 22 \\
Arthritis & $10.06 \%$ & 16 \\
Depression & $10.69 \%$ & 17 \\
Diabetes & $42.77 \%$ & 68 \\
Hypertension & $50.94 \%$ & 81 \\
Hypercholesterolemia & $25.79 \%$ & 41 \\
Gastritis & $7.55 \%$ & 12 \\
COPD & $16.35 \%$ & 26 \\
Chronic renal failure & $15.72 \%$ & 25 \\
Dementia & $3.77 \%$ & 6 \\
Parkinson disease & $1.26 \%$ & 2 \\
Autoimmune disease & $12.58 \%$ & 20 \\
Atrial fibrillation & $14.47 \%$ & 23 \\
Ischemic heart disease & $14.47 \%$ & 23 \\
Other cardiovascular disease & $10.06 \%$ & 16 \\
Ischemic heart disease & $6.92 \%$ & 11 \\
Other (specify) & $27.04 \%$ & 43 \\
\hline
\end{tabular}




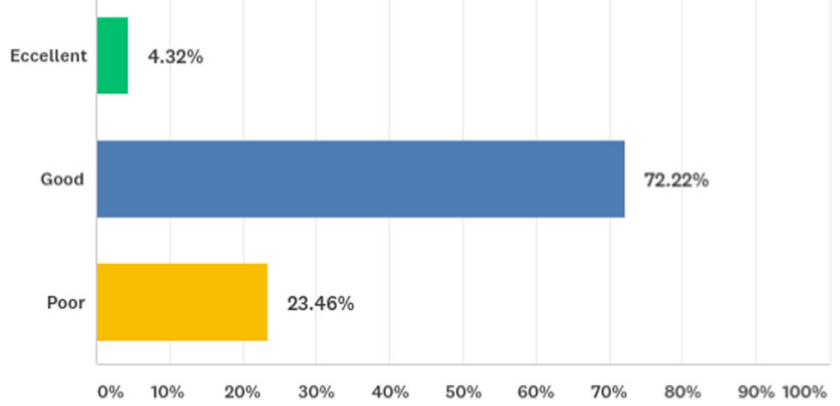

Fig. 2 Patients' health status

\section{Visit}

Eighteen percent of the 164 respondent physicians said they had spent more than 30 min on the visit. However, the majority admitted that they had not sufficiently investigated the wishes, needs, and state of health of the patient. In contrast, the majority (52\% of 163 respondents) devoted 5-10 min to the illustration of the prescription or advice, and most of the respondents spent 5-9 min to present and illustrate suggested educational materials or digital tools to the patient during the visit (Fig. 4).

Ninety-seven percent of the 156 respondent physicians stated they provided the patient with information on his/her condition during the visit. Still, only $36 \%$ of 155 respondents delivered educational material (brochures, leaflets, flyers).

\section{Empowerment of the patient}

Fifty percent of 154 respondents believe that patients should have an active role in caring for their health, but $76 \%$ out of 150 respondents believe that they should have just enough voice in making decisions regarding the management of their illness. The most widely used tool by doctors to actively involve the patient in managing and treating their health was dialogue (Fig. 5).

\section{Use of digital instruments and physician's experience}

Although the prescription of digital tools by the interviewed doctors is infrequent, most of the respondents who have used them are just satisfied enough with the results achieved by the patient with the use of the suggested digital tools (56\% of 139 respondents), while out of 142 respondents $68 \%$ agree or strongly agree that the use of digital tools for patient empowerment can improve health outcomes. More specifically, the respondent physicians agree that digital tools help educate and adequately inform patients about their pathology (63\%), educate and adequately inform patients about their treatments (59\%), have a better dialogue with them (45\%), improve adherence to therapy $(50 \%)$, increase patient compliance with their recommendations, tests, and prescriptions (47\%), and improve clinical practice (44\%). Few (39\% out of 142) physicians agree or strongly agree that the use of digital tools for patient empowerment reduces the time of the visit.

However, only $25 \%$ out of 142 respondents say that the use of digital tools on their patients and their clinical practice has had a "great positive effect." Most say it had a small positive effect $(58 \%)$ or no effect $(11 \%)$.

\section{Critical success factors}

According to the 142 respondents, clinical evidence could help to increase and improve the effectiveness of digital tools for patient empowerment, followed by involvement of the doctor in design, patient participation in design, reimbursement, and design features (Fig. 6).

Fig. 3 Physicians' prescription of digital tools

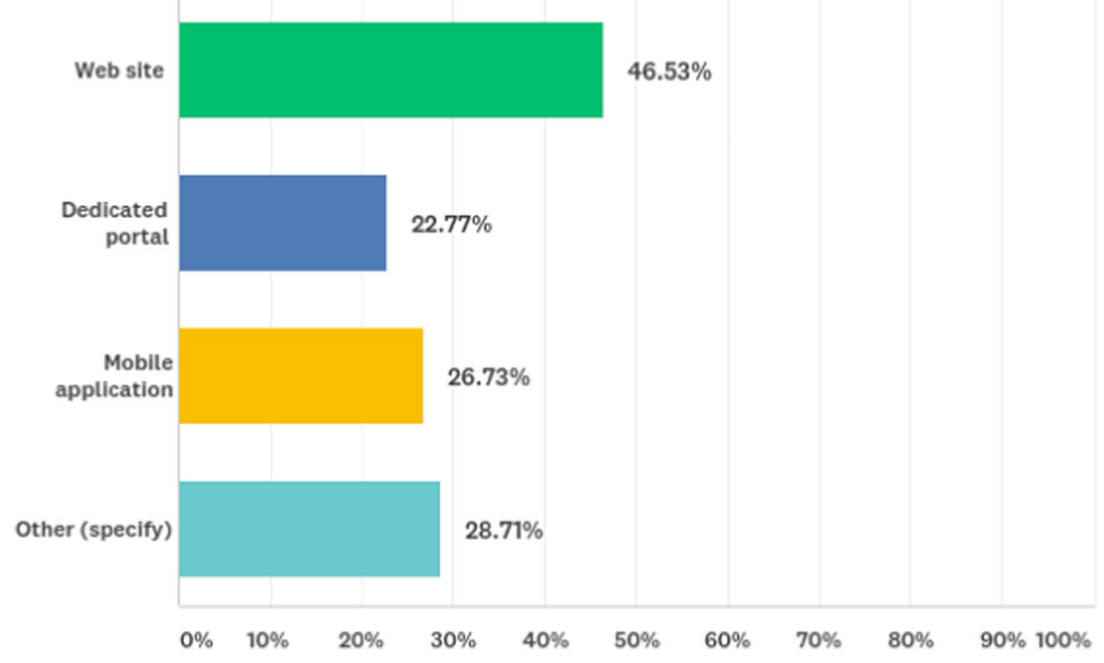


Fig. 4 Time spent presenting and illustrating suggested educational materials or digital tools to the patient during the visit

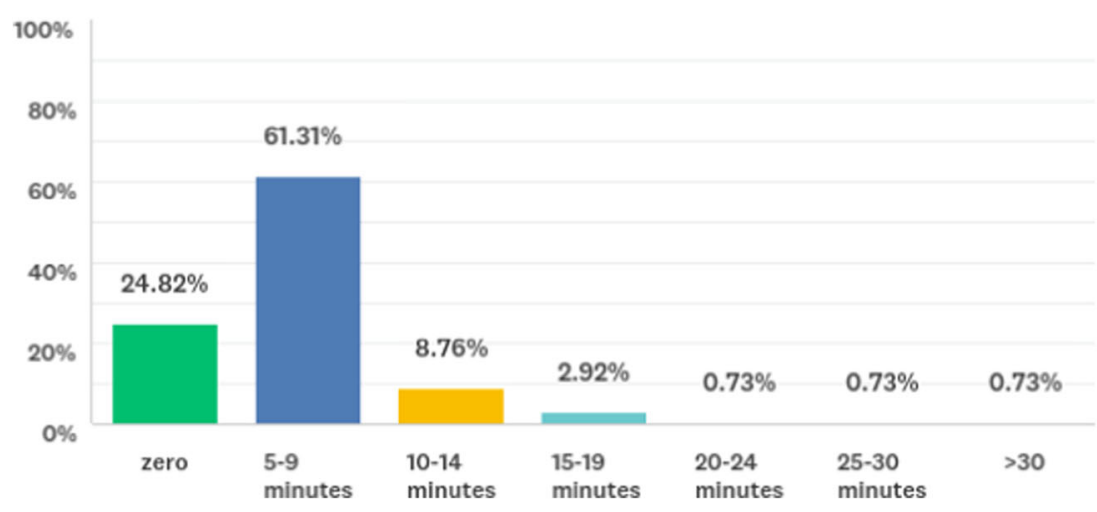

Proven clinical results (44\%) is indicated as the main missing element for the doctor to allow better and more effective empowerment of the patient, followed by:

- culture / forma mentis (35\%)

- support of the health organization (34\%)

- characteristics of the digital instrument based on the real needs of the patient (34\%)

- training (34\%),

- involvement of doctors in the design of digital tools $(32 \%)$,

- motivation (30\%),

- certification by institutions or scientific societies (24\%),

- characteristics of the digital tool based on the needs of the doctor (23\%), reimbursement (18\%),

- incentives (16\%).

\section{Discussion}

This study identifies several barriers and facilitators to the adoption of e-health technology by physicians to empower their patients. It provides a new perspective on the adoption of digital tools to empower patients.
One of the main themes that became apparent from our survey was the need for clinical evidence, which highlights how designing and implementing the right trials implies including not only the studied tools' clinical benefits but also the efficacy of the overall digital approach implemented in actual clinical settings. This was parallel with the results of the studies by Burke et al. (2015) and Fleming et al. (2020).

The second main theme that arose was patient/physician involvement in the design. This result was consistent with Faith Birnbaum et al.'s study (2015) which highlights how "existing digital health tools by-and-large fail to address the issues that matter to patients (Herz 2014) (and doctors Sullivan 2014), and fail to feel relatable" (IMS Institute for Healthcare Informatics 2013). To create relevant, usable, and effective digital health, it is critical to increase patients' involvement in the design of technological tools (Baker et al. 2014).

The third main theme that emerged in this study was the barriers surrounding training and support. This is related to the results of studies by de Grood et al. (2016), Boonstra and Broekhuis (2010), Castillo et al. (2010), Gagnon et al. (2010), and Goldstein et al. (2014), who support these findings.

Poor services from the vendor, such as inadequate training and support for problems associated with the e-health technology and poor follow-up, are barriers to the adoption of such devices. This situation is worsened by physicians' lack of
Fig. 5 Tools used by physicians to actively involve their patient in managing and treating her/his health

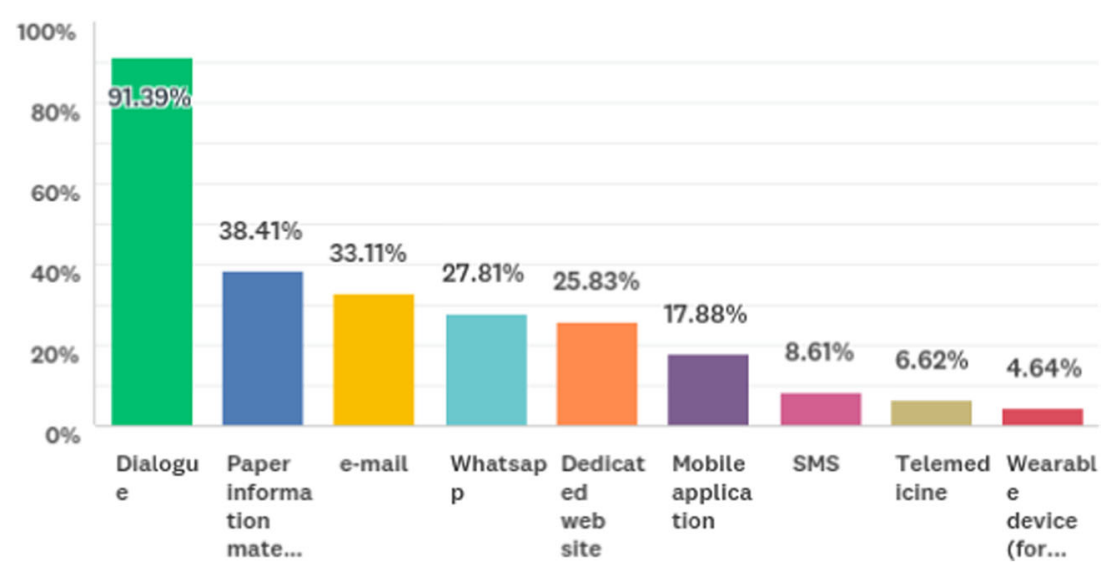


Fig. 6 Critical success factors to increase and improve the effectiveness of digital tools for patient empowerment

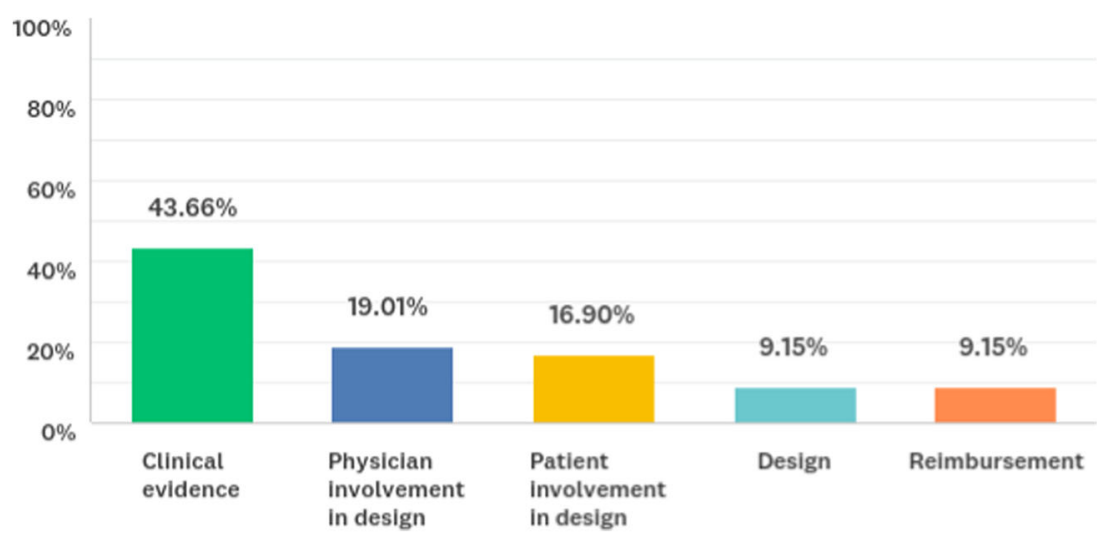

technical expertise and inherently complicated systems. To further facilitate the adoption of e-health technology, physicians need the technology tailored to the individuals' knowledge of e-health technology.

Furthermore, "on-site experts" who can provide first-line support are highly encouraged.

The fourth main theme that arose in this study was the way physicians used the time dedicated to the visit. According to Sinsky et al. (2016), in ambulatory care "for every hour physicians provide direct clinical face time to patients, nearly 2 additional hours is spent on EHR and desk work within the clinic day". In our survey, although most of the physicians reported dedicating a reasonable amount of time to the visit in the hospital, only a few of them dedicated enough time to illustrate materials provided and digital tools suggested to the patients. This can most probably be attributed to the business models and processes of both organizations, as well as a lack of training on both patient-physician communication and tools to be used in the new digital era. Needless to say that this puts at high risk the success of digital tools for patient empowerment.

\section{Conclusion}

The use of the internet for patient empowerment can potentially add value to people's health, to the clinical practice, and the overall healthcare system. Overcoming barriers to the widespread adoption of digital technologies could be a pivotal way to improve access to healthcare, and subsequently, health outcomes.

Our study adds to the current literature by showing that healthcare managers need to make sure that digital tools:

- are designed around physicians' and patients' needs,

- show clinical evidence of their benefits when introduced in the clinical context, and

- get enough attention from the internal organizations who must align their business models to the new needs and introduce the right tools and programs to support both physicians' and patients' adoption of new digital tools to pursue empowerment.

In this scenario, physician empowerment will be the crucial starting point to facilitate robust dialogue and exchange of information and mutual emotional support to complement the patient's rising authority. The present work offers an initial starting point for this endeavor.

\section{Future implications}

Successful organizations providing products and services in the healthcare industry have one thing in common: they are incredibly patient-centered and wholeheartedly committed to innovating both internally and externally.

For healthcare institutions and medical practices, successful patient empowerment relies not just on new technology but also a cultural shift. As the industry adapts to these changes, providers and healthcare administration must be prepared to face obstacles such as:

- difficulty shifting behaviors

- different communication preferences

- lack of health information exchanges

- technology ease of use

- operational and implementation challenges

- workforce reluctance

The many benefits of new healthcare technology and patient empowerment have been proven to outweigh the costs and difficulties of implementation. However, successful adaptation and cultural shifts rarely occur without obstacles. Also, one of the biggest challenges is the implementation of effective evidence-based methods of measurement for patient empowerment.

Patient empowerment has emerged as one of the most interesting keywords used by health politicians, academics, and practitioners in Europe and the US in recent years. However, 


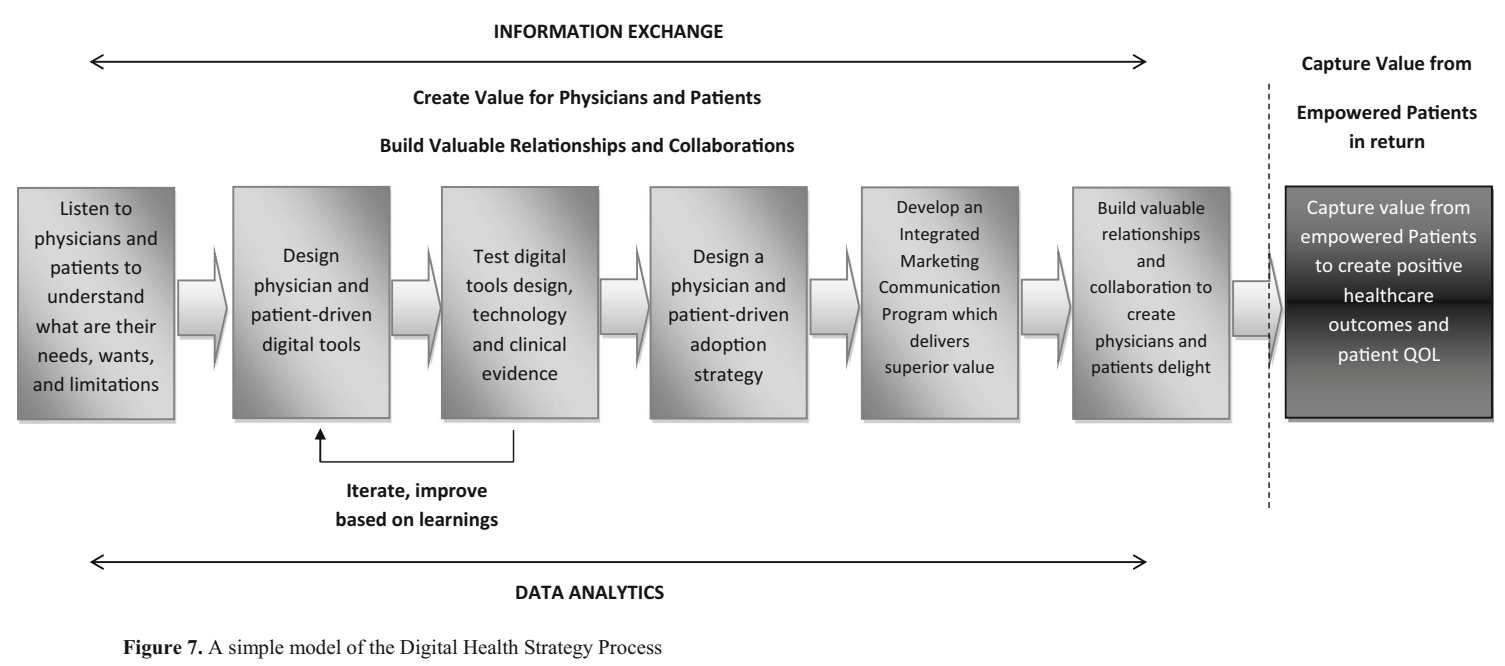

Fig. 7 A simple model of the Digital Health Strategy Process

to prove that it is more than a keyword, patient empowerment needs to be put into practice to demonstrate that it generates value. Most importantly, when dealing with new technologies, to effectively empower patients, we first need to focus on successfully empowering physicians. Overall, we should see empowerment as a philosophy or vision, as well as a strategy.

As a consequence, the Digital Health Strategy Process can be represented in a simple, seven-step model (Fig. 7) (Affinito 2019). In the first four steps, healthcare organizations act to understand physicians and patients, create physician and patient value, and build strong physician and patient relationships. In the final step, organizations bring back the rewards of creating superior value. In fact, by creating value for physicians and patients, they consequently capture value from patients in the form of empowerment, improved health, and healthcare outcomes.

Information exchange among all internal and external stakeholders and acquiring data for better decisions (data analytics) are two core aspects of this simple model.

\section{Methodological issues}

The data from our study raise at least two methodological questions about researchers 'ability to attribute specific motives to patients' empowerment.

First, patients rely on a multitude of both traditional and digital tools, and the process leading from disease diagnosis to a digital tool prescription and use in the clinical practice is very complicated.

Second, physicians who are interested in using digital tools to empower their patients may be more likely to exhibit better perception of their impact than others are, thus confounding the effect of health digital tools.

Thus, we expect prior interest in using health digital tools to be correlated with either age, physician's characteristics, and/or attention to and recall of the effect of awareness campaigns.

\section{Study limitations}

This investigation has certain limitations that may affect its interpretation and generalizability. The basic study design provides descriptive, cross-sectional data.

First, we did not collect information on outcomes for patients who had physician encounters.

Second, the national sample was too small to allow for rigorous control of underlying clinical conditions other than overall health status. Future studies restricted to specific conditions might obtain different results.

Third, samples are not representative of the entire European population of internists but represent an essential part of the reference populations. There was a heavy underrepresentation of physicians from most of the countries.

Fourth, as we surveyed hospital physicians we cannot generalize our results for ambulatory care. Main differences include organizational barriers, processes, and time allocation, while both settings share the same characteristics with regard to the lack of culture, training, a physician/patient-centered design and adoption management of digital tools for patient empowerment.

Acknowledgments The authors acknowledge FADOI (Italian Federation of the Associations of Hospital Internal Physicians) and EFIM (European Federation of Internal Medicine) for their unconditional and active support in endorsing the project and divulgating the online survey to their members.

Funding Information Open access funding provided by Università degli Studi di Milano within the CRUI-CARE Agreement.

\section{Compliance with ethical standards}

This study was conducted in accordance with the European Regulation 679/2016 (General Data Protection Regulation, known as GDPR) for the protection of human subjects, which regulates the processing of personal data and the rights and freedom of all subjects participating in research 
efforts. All study participants participated on a volunteer basis. No identifying information was collected or reported in this study. Additionally, participants benefited from human subject protection measures through their affiliation with the professional association that administered the study. As information related to anonymous and unidentified subjects does not fall within the scope of the aforementioned Regulation, no ethics committee review was required for this study.

Conflict of interest The authors certify that they have NO affiliations with or involvement in any organization or entity with any financial interest (such as honoraria; educational grants; participation in speakers' bureaus; membership, employment, consultancies, stock ownership, or other equity interest; and expert testimony or patent-licensing arrangements), or non-financial interest (such as personal or professional relationships, affiliations, knowledge or beliefs) in the subject matter or materials discussed in this manuscript.

Open Access This article is licensed under a Creative Commons Attribution 4.0 International License, which permits use, sharing, adaptation, distribution and reproduction in any medium or format, as long as you give appropriate credit to the original author(s) and the source, provide a link to the Creative Commons licence, and indicate if changes were made. The images or other third party material in this article are included in the article's Creative Commons licence, unless indicated otherwise in a credit line to the material. If material is not included in the article's Creative Commons licence and your intended use is not permitted by statutory regulation or exceeds the permitted use, you will need to obtain permission directly from the copyright holder. To view a copy of this licence, visit http://creativecommons.org/licenses/by/4.0/.

\section{References}

Affinito L (2019) Empowering the connected physician in the E-patient era: how physician's empowerment on digital health tools can improve patient empowerment and boost health (care) outcomes. Routledge, London

Ajami S, Ketabi S, Saghaeian-Nejad S et al (2011) Requirements and areas associated with readiness assessment of electronic health records implementation. J Health Admin 14:71-78

Anderson RM, Funnell MM (2010) Patient empowerment: myths and misconceptions. Patient Educ Couns 79(3):277-282. https://doi. org/10.1016/j.pec.2009.07.025

Aujoulat I, Marcolongo R, Bonadiman L, Deccache A (2008) Reconsidering patient empowerment in chronic illness: a critique of models of self-efficacy and bodily control. Soc Sci Med 66(5): 1228-1239. https://doi.org/10.1016/j.socscimed.2007.11.034

Baker T, Gustafson D, Shah D (2014) How can research keep up with eHealth? Ten strategies for increasing the timeliness and usefulness of eHealth research. JMIR 2014:16(2)

Birnbaum $F$ et al (2015) Patient engagement and the design of digital health. Acad Emerg Med 22(6):754-756. https://doi.org/10.1111/ acem. 12692

Boonstra A, Broekhuis M (2010) Barriers to the acceptance of electronic medical records by physicians from systematic review to taxonomy and interventions. BMC Health Serv Res 10:231

Burke LE, Ma J, Azar KMJ et al (2015) Current science on consumer use of mobile health for cardiovascular disease prevention a scientific statement from the American Heart Association. Circulation 132: 1157-1213. https://doi.org/10.1161/CIR.0000000000000232

Cahn A, Akirov A, Raz I (2018) Digital health technology and diabetes management. J Diabetes 10(1):10-17. https://doi.org/10.1111/17530407.12606

Calvillo J et al (2013) How technology is empowering patients? A literature review. Health Expect 18:643-652

Castillo V, Martinez-Garcia A, Pulido J (2010) A knowledge-based taxonomy of critical factors for adopting electronic health record systems by physicians: a systematic literature review. BMC Med Inform Decis Making 10:60

de Grood C, Raissi A, Kwon Y, Santana MJ (2016) Adoption of e-health technology by physicians: a scoping review. J Multidiscip Healthc 9:335-344. Published online 2016 Aug 1. https://doi.org/10.2147/ JMDH.S103881

Del Missier F, Ferrante D, Costantini E (2006) Focusing effects in predecisional information acquisition. Acta Psychol 125(2):155174

Fleming GA et al (2020) Diabetes digital app technology: benefits, challenges, and recommendations. A consensus report by the European Association for the Study of Diabetes (EASD) and the American Diabetes Association (ADA) Diabetes Technology Working Group. Diabetes Care 43(1):250-260. https://doi.org/10.2337/dci19-0062

Funnell MM, Anderson RM, Arnold MS, Barr PA, Donnelly M, Johnson PD et al (1991) Empowerment: an idea whose time has come in diabetes education. Diabetes Educ 17:37-41

Gagnon M, Desmartis M, Labrecque M et al (2010) Implementation of an electronic medical record in family practice: a case study. Inform Prim Care 18(1):31-40

Gibson CH (1991) A concept analysis of empowerment. J Adv Nurs 16(3):354-361. https://doi.org/10.1111/j.1365.2648.1991.tb01660. $\mathrm{x}$

Goldstein DH, Phelan R, Wilson R et al (2014) Brief review: adoption of electronic medical records to enhance acute pain management. Can J Anaesth 61(2):164-179

Goldzweig CL, Orshansky G, Paige NM et al (2013) Electronic patient portals: evidence on health outcomes, satisfaction, efficiency, and attitudes: a systematic review. Ann Intern Med 159:677-687

Herz JC (2014) Wearables are totally failing the people who need them most. Wired. Available from: http://www.wired.com/2014/11/ where-fitness-trackers-fail/. Accessed 04 June 2020

IMS Institute for Healthcare Informatics (2013) Patient apps for improved healthcare: from novelty to mainstream. https://developer.imshealth. com/Content/pdf/IIHI Patient Apps Report.pdf. Accessed 1 March 2015

Laverack G (2006) Using a 'domains' approach to build community empowerment. Commun Dev J 41(1):4-12. https://doi.org/10. 1093/cdj/bsi038

Mahmood A, Kedia S, Wyant DK, Ahn S, Bhuyan SS (2019) Use of mobile health applications for health-promoting behavior among individuals with chronic medical conditions. Digit Health 5:2055207619882181. Published 2019 Oct 10. https://doi.org/10.1177/2055207619882181

Milani RV, Lavie CJ, Bober RM, Milani AR, Ventura HO (2017) Improving hypertension control and patient engagement using digital tools. Am J Med 130(1):14-20. https://doi.org/10.1016/j. amjmed.2016.07.029

Monteagudo Peña JL, OM Gil (2009) E-health for patient empowerment in Europe. Ministero de Sanidad y Consumo, Madrid

Page N, Czuba CE (1999) Empowerment: what is it?. J Ext 37(5). http:// www.joe.org/joe/1999october/comm1.html 
Shahin I (2008) Managing the psychology of health care: what it means and what it is worth. McGill J Med 11(2):191-198

Sinsky C, Colligan L, Li L et al (2016) Allocation of physician time in ambulatory practice: a time and motion study in 4 specialties. Ann Intern Med 165(11):753-760. https://doi.org/10.7326/M16-0961

Sleurs K, Seys SF, Bousquet J et al (2019) Mobile health tools for the management of chronic respiratory diseases. Allergy 74(7):12921306. https://doi.org/10.1111/all.13720

Sullivan M (2014) Guess what? Doctors don't care about your FitBit data. VentureBeat News. Available from: http://venturebeat.com/2014/ 08/15/guess-what-doctors-dont-care-about-your-fitbit-data/. Accessed 04 June 2020
Tverksy A, Kahneman D (1973) Availability: a heuristic for judging frequency and probability. Cogn Psychol 4:207-232

Walter Z, Lopez MS (2008) Physician acceptance of information technologies: role of perceived threat to professional autonomy. Decis Support Syst 46:206-215

World Health Organization (2009), WHO guidelines on hand hygiene in health care: first global patient safety challenge clean care is safer care. WHO, Geneva. Available at https://www.ncbi.nlm.nih.gov/ books/NBK144022/. Accessed 04 June 2020

Publisher's note Springer Nature remains neutral with regard to jurisdictional claims in published maps and institutional affiliations. 